\title{
Do Hummingbirds See in Ultraviolet?
}

\author{
M. Curé*,1 and A.G. Palacios ${ }^{2}$ \\ ${ }^{I}$ Departamento de Física y Astronomía, Facultad de Ciencias, Universidad de Valparaíso, Chile \\ ${ }^{2}$ Centro de Neurociencia de Valparaíso, Facultad de Ciencias, Universidad de Valparaíso, Chile
}

\begin{abstract}
We present a numerical model to fit the electroretinogram (ERG), a gross evoked eye visual potential, that originate in the retina through photons absorption by photoreceptors and then involve the contribution form others retinal neurons. We use the ERG measured in a hummingbird, to evaluate the most likely retinal mechanism - cones visual pigments and oil-droplets - that participate in their high dimensional tetra or pentachromatic color hyperspace. The model - a nonlinear fit - appears to be a very useful tool to predict the underlying contribution visual mechanism for a variety of retinal preparation.
\end{abstract}

Keywords: Color vision, electroretinogram, non lineal model.

\section{INTRODUCTION}

A critical question in visual sciences is to determinate the types of photoreceptors that contribute - for a particular eye to the overall retinal spectral sensitivity. We have developed a mathematical model that helps to answer this question. As a case study, we have used the electroretinogram results of a diurnal bird, the Firecrown hummingbirds. It has been well established that hummingbirds learn to associate the rich nectar contents of flowers with different colors, varying from red to ultraviolet [1-6] and it is of particular interest to know the neural bases for such chromatic properties. Avian retinae show a complex array of photoreceptors, including single and double cones with visual pigments of maximal absorption sensitivity $\left(\lambda_{\max }\right)$ at long-wavelength-sensitive cone visual pigment (L-cone) with $\lambda_{\max }$ between $540 \mathrm{~nm}$ and $570 \mathrm{~nm}$; mediumwavelength-sensitive cone visual pigment (M-cone) with $\lambda_{\max }$ between $500 \mathrm{~nm}$ and $507 \mathrm{~nm}$; short-wavelength-sensitive cone visual pigment (S-cone) with $\lambda_{\max }$ between $430 \mathrm{~nm}$ and $460 \mathrm{~nm}$ and violet-wavelength-sensitive cone (V-cone) with $\lambda_{\max }$ (between $400 \mathrm{~nm}$ and $420 \mathrm{~nm}$ ) or ultraviolet-sensitivecone (UV-cone) with $\lambda_{\max }$ (between $360 \mathrm{~nm}$ and $370 \mathrm{~nm}$ ) [79]. A special trait of bird's cones is the presence of oil-droplets spherical lipid structures inserted in the inner segment of the photoreceptor - which form long pass filters with optical properties that depend on carotenoid contents and concentrations [8] (also see section 2.1.2 below). The V-cone or UV-cone contain a transparent (T) oil-droplet with no carotenoid; the Scones a galloxanthin (pale appearance); the M-cones a zeaxanthin (yellow appearance); and L-cones an astaxanthin (red) in

*Address correspondence to this author at the Departamento de Física y Astronomía, Facultad de Ciencias, Universidad de Valparaíso, Chile. Gran Bretaña 1111, Playa Ancha, Valparaíso, Chile; E-mail: michel.cure@uv.cl high concentrations. Double cones have L visual pigments and are screened by a variety of galloxanthin and $\varepsilon$-carotene mixtures $[2,8,10-12]$. The final cone mechanism sensitivity is then determined by combining the cone visual pigment absorption and oil-droplet transmittance. In many birds, ultraviolet (UV) is a color that is believed to be involved in social communication, food or sexual selection and it is of particular interest to know their presence in many vertebrates' species [13]. We developed a theoretical non-linear model that takes into account the additive - the assumed mode here - combination of the spectral properties of different cones visual pigments and their oil-droplets complements. Here we compare the predictions of this model with the electroretinogram (ERG), a gross retina visual evoke potential, response outcome.

\section{THE ELECTRORETINOGRAM (ERG)}

All the details of the experimental work are described in detail in $[14,15]$. In brief, the optical system consisted of a stabilized power supply with a quartz lamp (250W, ORIEL), a monochromator (1200 lines $\mathrm{mm}^{-1}$ grating, ORIEL, $20 \mathrm{~nm}$ half-bandwidth). A short-pass filter (UG11) to isolate the UV band and long-pass filters (SCHOTT $R G 500, R G 540$, $R G 680$ ) to eliminate stray light from the monochromator, were used. An electronic shutter (Uniblitz, Vincent Associates) was utilized to control flash duration and an optical quartz wedge ( $0-5$ Optical Density) was inserted to attenuate the flash intensity. The monochromator, optical wedge and shutter were under computer control and adjusted to deliver short flashes at wavelengths from 300 to $700 \mathrm{~nm}$ in $20 \mathrm{~nm}$ steps. The eye was kept light adapted by a quartz tungsten lamp $(150 \mathrm{~W})$ producing a background illumination of $240 \mu \mathrm{W} / \mathrm{cm}^{2} \mathrm{sr}$ at the cornea. The light stimulus was collimated at the cornea using a quartz condenser. After general anesthesia the ERG signal was recorded using a pair of ring $\mathrm{Ag} / \mathrm{AgCl}$ electrodes, one placed on the cornea and the other - reference electrode - under the head skin, and 
amplified with a high gain amplifier (DP-301 Warner Instruments). The ERG spectral sensitivity function $S(\lambda)$ was calculated from $r_{\text {peak }} / I$; where $I$ is the flash photon flux, and $r_{\text {peak }}$ is the b-band peak of the ERG response, evoked by a series of dim flashes $(n=50-100)$. Fig. (1) illustrates a theoretical ERG response, where the $a$-band responses correspond to the photoreceptors input and the $b$-band is related to a class of retinal $\mathrm{ON}$ bipolar cells.

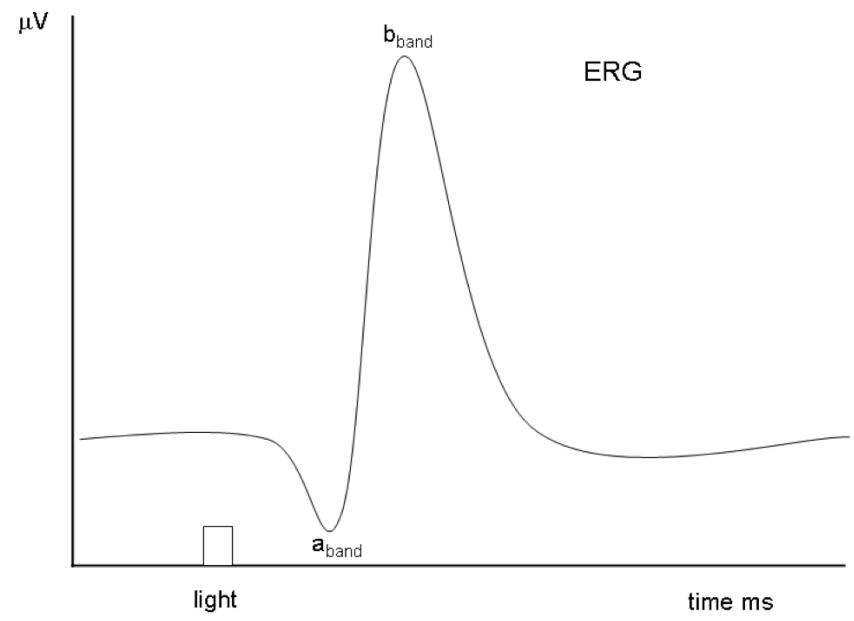

Fig. (1). Simulated ERG example showing the different characteristic a-band and b-band evoked by a flash light stimulation.

\subsection{The Pigment's Functions}

\subsubsection{The Primary Pigment Function}

The photochemistry of visual pigments in the $300-700$ $n m$ spectral range shows two principal absorption $\alpha_{b a n d}$ and $\beta_{\text {band }}$ peaks. The spectral sensitivity of $\alpha_{b a n d}$ depends on the absorption properties of a chromophore (11-cis retinal) covalently linked to an opsin protein, and follows [16], namely:

$$
P\left(\lambda, \lambda_{\max }\right)=e^{A\left(a-\frac{\lambda_{\max }}{\lambda}\right)}+e^{B\left(b-\frac{\lambda_{\max }}{\lambda}\right)}+e^{C\left(c-\frac{\lambda_{\max }}{\lambda}\right)}+D^{-1}
$$

where $a$ is the visual pigment dependence as function of the wavelength:

$a=0.8795+0.0459 e^{\left(\lambda_{\max }-300\right)^{2} / 11940}$

and $\lambda_{\max }$ refers to the peak absorption. The others constants are: $A=69.7, B=28 ., C=-14.9, D=0.674, b=0.922$, $c=1.104$. The $\beta_{\text {band }}$ absorption can be estimated using a log-normal function [17] and $\beta_{\lambda_{\max }}$ location is related to $\alpha_{\lambda_{\max }}[18]$ by:

$\beta_{\lambda_{\max }}=0.429 \alpha_{\lambda_{\max }}+123$

Finally, the visual pigments are given by:

$P_{i}=\alpha_{\text {band }}+u_{i} \beta_{b a n d}$, where $u_{i}$ represent the relative amplitude of the $\beta_{b a n d}$. As an example, we show in Fig. (2) the absorption properties of a visual pigment with $\lambda_{\max }=510 \mathrm{~nm}$ and $u_{i}=0.26$

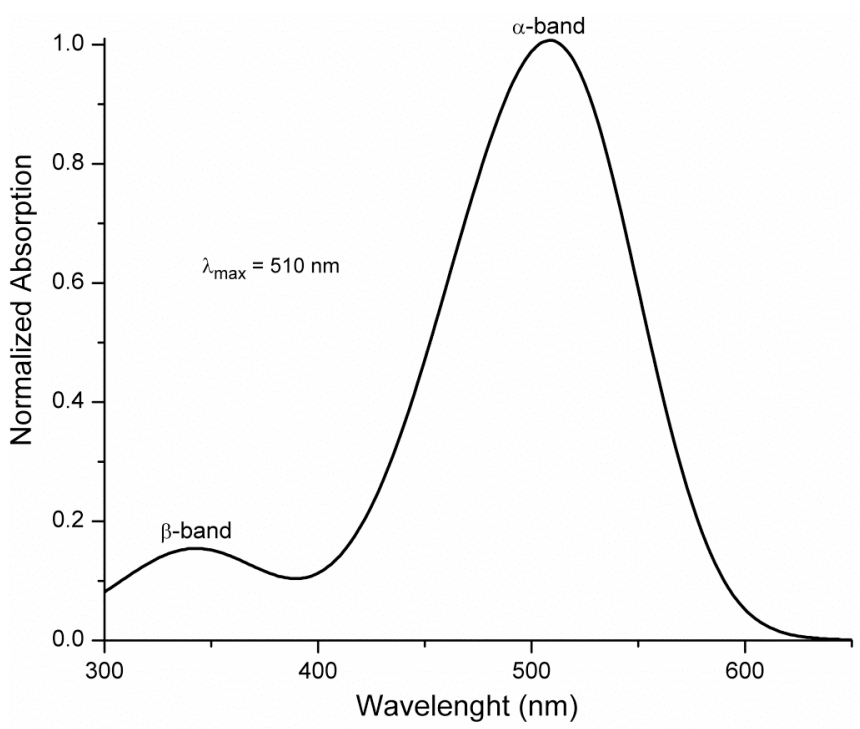

Fig. (2). Visual pigment template with $\lambda_{\max }=510 \mathrm{~nm}$ and $u_{i}=0.26$, calculated using Eqs. 1 to 4 .

\subsubsection{The Oil-Droplet Filters Function}

The filter effect of an oil-droplet is given by:

$$
S(\lambda)=10^{-c^{\prime} D^{\prime}(\lambda)}
$$

where $D^{\prime}(\lambda)$ corresponds to the normalized absorbance spectra of the carotenoid and $c^{\prime}$ its peak absorbance (see [19]). For an analytical method in estimating the oil-droplets filter properties see [2].

\section{THE VISUAL PIGMENTS AND THE NON-LINEAR MODEL}

According to the above, the spectral response of the ERG can be reproduced by:

$\phi(\lambda)=\sum_{i=1}^{n} k_{i} S_{i}(\lambda) P_{i}\left(\lambda, \lambda_{\max }\right)$

where $n=5$ is the number of different cones in avian retina, $i$ is corresponding index, $k_{i}$ the relative contribution of different chromatic mechanisms, $P_{i}$ is the absorbance spectra of cones (Eq. 4) and $S_{i}$ is the associated oil-droplets (Eq. 5).

\subsection{Parameters Constraints}

Before performing fits, we constrain our model by defining ranges of values for certain parameters. The used restrictions are:

- $\quad k_{i} \geq 0$, this is valid for all $k_{i}$ because the contribution to the total sensitivity must be positive. 
- $\quad 0 \leq c_{c^{\prime}}{ }_{i} \leq 20$, this restriction is related to each of oildroplets pigment concentration associated to each photoreceptor.

- $0.1 \leq u_{i} \leq 0.6$ for the $\beta_{\text {band }}$ amplitude due to observed values in the literature [18].

- $-5 \leq \lambda_{i} \leq 5$ is valid for all $\lambda_{\max }$ of the $\alpha_{\text {band }}$ of different types of cones pigments.

\section{THE NON-LINEAR FIT}

We want to minimize the following least-square function:

$\Psi=\sum_{k=1}^{N}\left(\phi\left(\lambda_{k}\right)-y_{k}\right)^{2}$

where $y$ represents the measured values of the ERG spectral sensitivity and $N$ gives the number of measured data points.

The algorithm we used to find a minimum of this function is the Nelder \& Mead downhill simplex algorithm [20]. In order to find the global minimum and not just a local minimum, we run the program many times $(n=500)$ starting each time with a different set of randomly selected values for the initial parameters.

This procedure give us the global minimum $\left(\Psi_{\min }=0.06347\right)$ and we proceed to compare the measured data $y$ with the fitted data $\phi$ obtained using the parameters of the global minimum. Fig. (3) shows $y$ versus $\phi$, the continuous straight-line shows the linear relationship between them. In view of this linear relationship, we calculated the Pearson correlation index for each one of the runs that we performed. Fig. (4) shows all the minimae founded in our runs plotted against their correlation index.

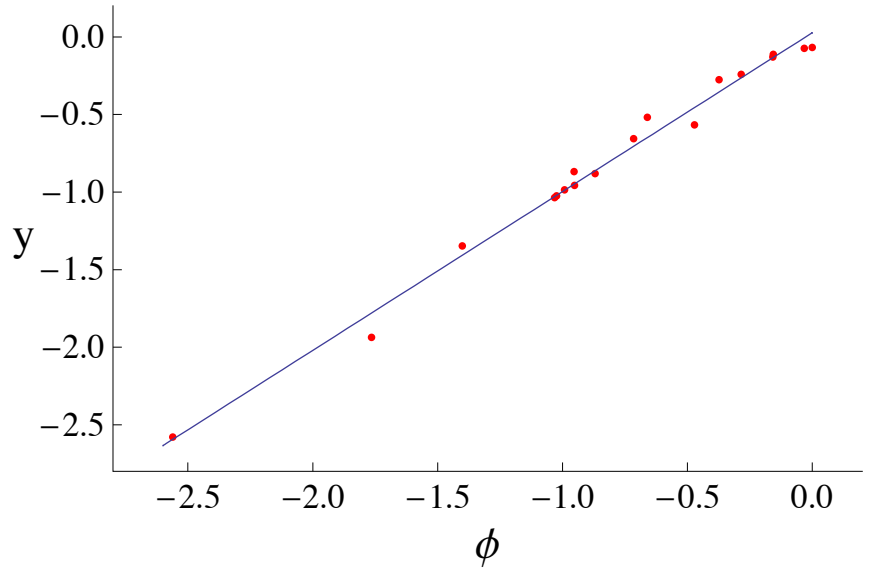

Fig. (3). The measured data $y$ versus the fitted data $\phi$. There clearly exhibit a linear relationship.

The last plot shows clearly that the best fit corresponds to the best Pearson's correlation, the value of this one is $R=0.9942$. Fig. (5) shows the measured ERG data along with the best fit obtained $\phi$ (solid black line) and the relative contribution of each of the visual mechanisms involved (see Eq. 6). Table 1 shows the numerical data illustrated in Fig. (5) and reflects the best fit model.

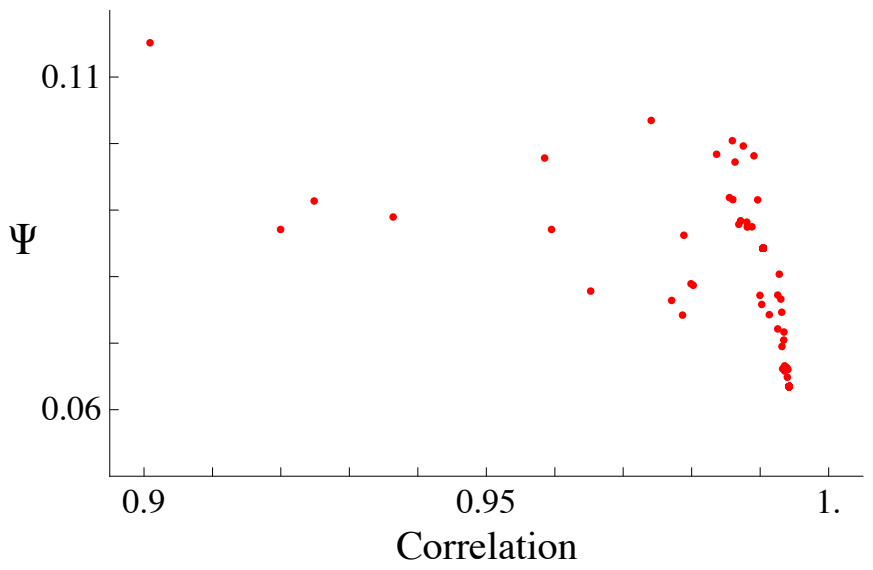

Fig. (4). This plot shows all the minima values of $\Psi$ versus the corresponding Pearson's correlation. The Global minimum is the one with the best correlation.

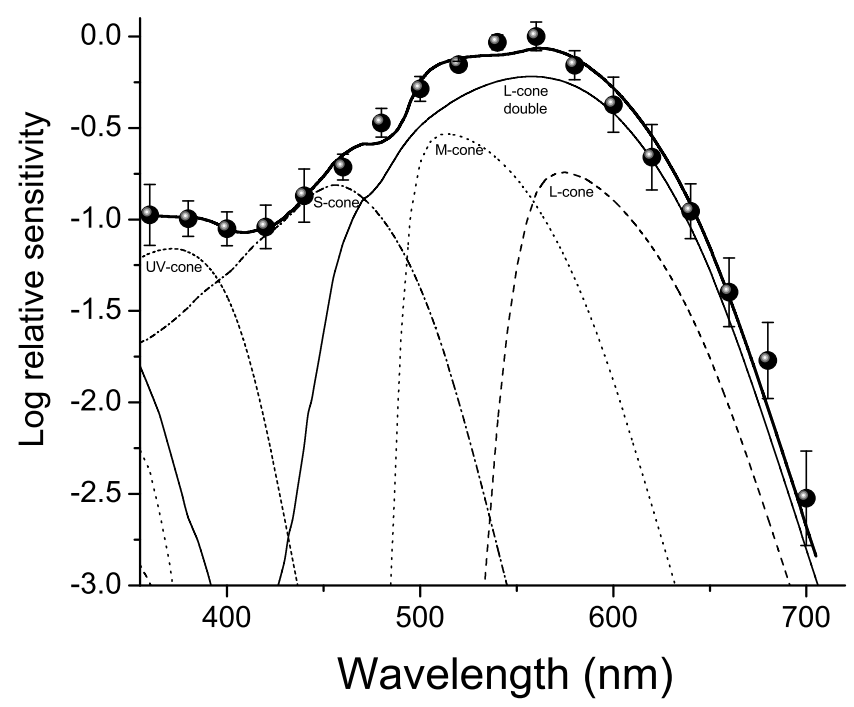

Fig. (5). The contribution of each visual mechanism, the total spectral sensitivity and the measured data (see text for details and Table 1 for relative values).

Table 1.

\begin{tabular}{|c|c|c|c|}
\hline $\begin{array}{c}\text { Visual } \\
\text { Pigment }\end{array}$ & $\begin{array}{c}\lambda_{\max } \\
(\mathbf{n m})\end{array}$ & $\begin{array}{c}\text { Carotenoid } \\
\text { (Optical Density) }\end{array}$ & $\begin{array}{c}\text { Relative } \\
\text { Contribution }\end{array}$ \\
\hline \hline L double cone & 560 & 2.0 & $45 \%$ \\
L single cone & 560 & 8.0 & $15 \%$ \\
M cone & 508 & $3.0(\mathrm{CisC})$ & $22 \%$ \\
& & $5.0($ TransC) & \\
S cone & 444 & 0.3 & $13 \%$ \\
UV cone & 371 & - & $5 \%$ \\
\hline
\end{tabular}

\section{CONCLUSION}

We model the photopic ERG sensitivity of an avian retina as a function of four singles cones and one double cone mechanism (visual pigment + oil-droplets). In order to get a 
global minimum we run the simulation process 500 times starting each time with a different random seed. The global minimum that we find corresponds to the highest Pearson correlation coefficient between the ERG data and the best fit obtained. From the best fit we find $\lambda_{\max }$ at $371 \mathrm{~nm}$ for the UV-cone; $444 \mathrm{~nm}$ for the S-cone; $508 \mathrm{~nm}$ for the M-cone and $560 \mathrm{~nm}$ for the L-cone (see also Table 1). This result suggests the contribution of five different cone pigments, including one into the UV. One point of precaution is that a definitive proof of the presence of those particular cones mechanism should be confirmed by complementary studies (e.g. Microspectrophotometry, behavioral data; cloning of visual protein genes and its expression absorbance measures in vitro). In general, it has been described that double cones dominate in avian retina (close to $50 \%$ of the all types of cones) and their contribution to the ERG is essential and can mask the contribution of other cone mechanisms. While our results confirm double cone contributions they also suggest the participation of four single cone mechanisms. To the best of our knowledge, this is the first work using a numerical model to analyze the ERG, a relative general measure of the eye visual function, by combining contributions of individual cone mechanisms. In recent experiments, we have extended our model application to a trichromatic color vision insect with very promising predictions [21].

An important limitation of the present version of the model is the assumption that the retinal signal of individual mechanism can simply be integrated according to additives rules. As an important neural property, for example, the presence of opponent (subtractive) mechanisms shows vertebrates retina to be involved [22]. Furthermore, we must look to the diversity array of cones in function of behavioral experiments to further explore models to explain color vision in birds $[23,24]$.

\section{ACKNOWLEDGEMENTS}

We thank Servicio Agricola Ganadero (SAG) permission \# 1392 for animal captures. Animal care and experimental procedures complied with international regulations (NIH publications Number 80-23). Funding by Fondecyt \#1000396 (AGP) and PBCT-CONICYT ACT45 to AGP. Finally, we want to thank M.R. Schreiber for his valuable comments and language help in improving this manuscript.

\section{REFERENCES}

[1] Waser NM, Chittka L, Price MV, Williams N, Ollerton J. Generalization for pollination systems, and why it matters. Ecology 1996; 77: $1043-60$

[2] Vorobyev M. Coloured oil droplets enhance colour discrimination. Proc Biol Sci 2003; 270: 1255-6.
[3] Huth HH, Burkhardt D. Der spektrale Sehbereich eines ViolettohrKolibris. Naturwissenschaften 1972; 59: 650.

[4] Goldsmith TH, Collins JS, Perlman DL. A wavelength discrimination function for the hummingbird Archilochus alexandri. J Comp Physiol 1981; 143: 103-10.

[5] Campbell B, Lack E. A dictionary of birds. Buteo Books, Vermillion, SD, USA 1985

[6] Altshuler DL. Flower color, hummingbird pollination, and habitat irradiance in four neotropical forests. Biotropica 2003; 35: 344-55

[7] Hart NS. The visual ecology of avian photoreceptors. Prog Retin Eye Res 2001; 20: 6757-703.

[8] Hart N, Vorobyev M. Modelling oil droplet absorption spectra and spectral sensitivities of bird cone photoreceptors. J Comp Physiol A 2005; 191: 381-92.

[9] Hart NS, Hunt DM. Avian visual pigments: characteristics, spectral tuning, and evolution. Am Nat 2007; 169: S7-S26.

[10] Goldsmith TH, Collins JS, Licht S. The cone oil-droplets of avian retinas. Vision Res 1984; 24: 1661-71.

[11] Bowmaker JK, Heath LA, Wilkie SE, Hunt DM. Visual pigments and oil droplets form six classes of photoreceptor in the retinas of birds. Vision Res 1997; 37: 2183-94.

[12] Vorobyev M, Osorio D, Bennett ATD, Marshall NJ, Cuthill IC. Tetrachromacy, oil droplets and bird plumage colours. J Comp Physiol A 1998; 183: 621-33.

[13] Hunt S, Cuthill IC, Bennett ATD, Church SC, Partridge JC. Is the ultraviolet waveband a special communication channel in avian mate choice? J Exp Biol 2001; 204: 2499-507.

[14] Herrera G, Zagal JC, Diaz M, et al. Spectral sensitivities of photoreceptors and their role in colour discrimination in the Greenbacked Firecrown Hummingbird (Sephanoides sephaniodes). J Comp Physiol A 2008; 194: 785-94.

[15] Herrera G, Fernández MJ, Pohl N, Diaz M, Bozinovic F, Palacios AG. Sistema Visual en el Colibri- Austral (Sephanoides sephaniodes) y el Picaflor Cordillerano (Oreotrochilus leucoplerus: Electroretinografía y Coloración. Ornitol Neotrop 2004; 15: 215-22.

[16] Govardovskii V, Fyhrquist N, Reuter T, Kuzmin D, Donner K. In search of the visual pigment template. Vis Neurosci 2000; 17: 50928.

[17] Stavenga DG, Smits RP, Hoenders BJ. Simple exponential functions describing the absorbance bands of visual pigment spectra. Vision Res 1993; 33: 1011-7.

[18] Palacios AG, Srivastava R, Goldsmith TH. Spectral and polarization sensitivity of photocurrents of amphibian rods in the visible and ultraviolet. Vis Neurosci 1998; 15: 319-31.

[19] Goldsmith TH, Butler BK. The roles of receptor noise and cone oil droplets in the photopic spectral sensitivity of the budgerigar, Melopsittacus undulatus. J Comp Physiol A 2003; 189: 135-42.

[20] Nelder J, Mead R. A simplex method for function optimization. Comp J 1965; 7: 308.

[21] Martinez J, Palacios AG, Marquez IN, Arroyo MTK, Mpodozis J. Can red flowers be conspicuous to bees? Bombus dahlbomii and South American temperate forests flowers as a case in point. Summited 2009

[22] Hughes A, Saszik S, Bilotta J, Demarco PJ Jr, Patterson WF 2nd. Cone contributions to the photopic spectral sensitivity of the zebrafish ERG. Vis Neurosci 1998; 15: 1029-37.

[23] Varela FJ, Palacios AG, Goldsmith TH. Color Vision of Birds. In Zeigler HP, Bischof HJ, Eds. Vision, brain, and behavior in birds. MIT Press Cambridge, MA 1993.

[24] Thompson E, Palacios A, Varela F. Ways of coloring: Comparative color vision as case study for cognitive science. Behav Brain Sci 1992; 15: 1-74. 